\title{
DISKUSSION
}

\section{Zur Verantwortung der Landtage für die europäische Integration*}

\author{
Hans-Jürgen Papier
}

\section{Das Parlamentarische System}

Die demokratische Ordnung des Grundgesetzes und der Verfassungen der Bundesländer ist eine dezidiert parlamentarische Demokratie; das Regierungssystem der Bundesrepublik ist ein dezidiert parlamentarisches Regierungssystem. Der Deutsche Bundestag ist auf der Ebene des Bundes das einzige Verfassungsorgan, das über eine unmittelbare Legitimation durch das Staatsvolk verfügt; in derselben Weise sind es in den Ländern, und zwar ausnahmslos und ausschließlich, die Landesparlamente, die unmittelbar vom Volk gewählt werden. Sämtliche anderen Verfassungsorgane in Bund und Ländern leiten ihre Legitimation von den Parlamenten ab. Die Parlamente sind notwendige Glieder in jeder demokratischen Legitimationskette, gleichgültig ob es organisatorisch-personell um die Berufung in staatliche Ämter oder ob es sachlich-inhaltlich um die Kontrolle und Verantwortlichkeit bei der Ausübung von Staatsgewalt geht. Die Parlamente in Bund und Ländern verfügen regelmäßig über das Gesetzgebungsmonopol, der rechtsstaatliche Vorbehalt des Gesetzes verlangt, dass die Parlamente selbst die wesentlichen Entscheidungen für das Gemeinwesen treffen. Die Wahrung substantieller parlamentarischer Zuständigkeiten und Befugnisse bildet neuerdings auch einen der Dreh- und Angelpunkte bei der Kontrolle des Prozesses der europäischen Integration. Die weiteren klassischen Funktionen der Parlamente, etwa das Budgetrecht, müssen an dieser Stelle nicht weiter ausgeführt werden.

Die Macht der Parlamente in Deutschland könnte also - jedenfalls auf dem Papier kaum größer sein. Deutschland hat ein Repräsentationssystem, das de jure stark von einem Parlamentsmonismus geprägt ist. Dennoch ist immer wieder von der Ohnmacht der Parlamente und von der „Entparlamentarisierung“ der Politik die Rede - und nicht ganz ohne Grund und ohne dass sich heftiger Widerspruch regen würde.

\section{Die Tendenz der „Entparlamentarisierung“}

Die partielle Kompetenzverlagerung von den Parlamenten auf die Regierungen ist zu einem gewissen Teil in der Funktionsweise der Bundesstaatlichkeit, also des Exekutiv- oder Beteiligungsföderalismus, vorgezeichnet. Zum Teil hat sich der Bedeutungsverlust der Parlamente vielleicht auch nur in der Praxis eingeschlichen. Betrachtet man das Verhältnis von parlamentarischer Demokratie und bundesstaatlicher Gliederung, wird man berücksichtigen

* Vortrag auf der Jahreskonferenz der Präsidentinnen und Präsidenten der deutschen Landesparlamente, Stuttgart, 21. Juni 2010. 
müssen, dass Föderalismus nicht nur eine simple Vervielfachung der Parlamente - im Bund, in den Ländern und auf der europäischen Ebene - bedeutet. Aus der Verbindung von föderativem und demokratischem Verfassungsprinzip ergibt sich vielmehr eine qualitative Veränderung des politischen Systems, in der eine Bedeutungseinbuße der Parlamente zum Teil in der Konstruktion angelegt ist.

Schon seit längerem ist zu konstatieren, dass sich Rechtsetzung zunehmend von den niedrigeren auf höhere Ebenen verlagert. Das gilt sowohl für das Verhältnis zwischen Bund und Ländern als auch für das Verhältnis zwischen der Europäischen Union und ihren Mitgliedstaaten. Weder das Subsidiaritätsprinzip noch - innerstaatlich - die Bedürfnis- beziehungsweise Erforderlichkeitsklausel des Art. 72 Abs. 2 GG haben bislang einen wirksamen Schutz zugunsten der jeweils niedrigeren Regelungsebene gewähren können. Mit dieser „Hochzonung“ von Zuständigkeiten hat aber nicht eine bloße Aufgabenverlagerung zwischen den Parlamenten stattgefunden, also eine Art „In-sich-Geschäft“ der Parlamente untereinander. An die Stelle der verloren gegangenen Parlamentszuständigkeiten auf der jeweils niedrigeren Ebene sind Beteiligungsrechte der Exekutive an der Rechtsetzung auf der höheren Ebene getreten. Sehr plastisch wird deshalb insoweit auch von „Beteiligungs-“ oder „Exekutivföderalismus“ gesprochen.

Innerstaatlich handelt es sich um die Mitwirkung der Länder an der Gesetzgebung des Bundes durch den Bundesrat, der bekanntlich aus Mitgliedern der Landesregierungen besteht; im Rahmen der Europäischen Union bildet die zentrale Rechtsetzungsinstanz ein Rat, der sich aus Vertretern der Mitgliedstaaten auf Ministerebene zusammensetzt. In beiden Fällen sind es also Regierungsvertreter, die an Stelle des Parlaments agieren; und in beiden Fällen handelt es sich nicht mehr um Entscheidungen, die für die jeweilige politische Einheit autonom getroffen werden, sondern die in zwischenstaatlicher beziehungsweise intergouvernementaler Kooperation einheitlich für eine Mehrzahl von Staaten ausgehandelt und - jedenfalls vielfach - nach dem Mehrheitsprinzip beschlossen werden.

\section{Betroffenheit der Landesparlamente}

Durch die zunehmende Verlagerung von Rechtsetzungskompetenzen auf die Unionsebene haben in der föderalen Ordnung Deutschlands vor allem die Landesparlamente einen zusätzlichen Bedeutungs- und Kompetenzverlust hinnehmen müssen, denn in dem Prozess der zunehmenden Vergemeinschaftung von Regelungsbereichen sind seit geraumer Zeit und mit dem Wirksamwerden des Lissabon-Vertrages noch in verstärktem Maße - Felder der klassischen Innenpolitik wie die der öffentlichen Sicherheit, Bildung, Kultur, Medien und öffentlichen Daseinsvorsorge betroffen, die in der bundesstaatlichen Ordnung Deutschlands an sich in die Gesetzgebungskompetenzen der Bundesländer fallen. Rechtssätze der Union werden auf diesen Feldern nicht nur in unmittelbarer Mitwirkung allein der Gubernative des Bundes erlassen; auch die grundgesetzlich vorgesehenen innerstaatlichen Mitwirkungsrechte sind auf die Gesetzgebungsorgane des Bundes beschränkt. Die deutschen Bundesländer sind in dem in Art. 23 Abs. 2 bis 7 GG geregelten Mitwirkungsprozess um ein weiteres Mal mediatisiert, weil es nach der bundesstaatlichen Verfassungsordnung der Bundesrat ist, der gewissermaßen als Treuhänder für die Länder diese Mitwirkungsrechte wahrnimmt. Von Bundesverfassung wegen bleiben die Landesparlamente in diesem Beteiligungsverfahren außen vor. 
Eine entsprechende Mediatisierung der Bundesländer ist bei der Durchsetzung des Subsidiaritätsprinzips vorgesehen, das als materielles Prinzip schon bislang im primären Gemeinschaftsrecht verankert gewesen war, nun aber durch das Lissaboner Vertragswerk vor allem eine prozedurale Stärkung erfahren soll. Durch das so genannte Frühwarnsystem und die Subsidiaritätsklage werden die Parlamente und ihre einzelnen Kammern zum wahren Hüter des Subsidiaritätsgedankens erkoren. Das hat auch eine gewisse Logik, sind es doch gerade die nationalen Parlamente, die die Verlierer im Prozess der zunehmenden Verlagerung der Rechtsetzung auf die Unionsebene sind. Eine solche Zuständigkeit stärkt zwar in einer bundesstaatlichen Ordnung wie der Deutschlands tendenziell die Stellung der Bundesländer, aber auch hier sind Subsidiaritätsrüge und Subsidiaritätsklage von Bundesrechts wegen insoweit dem Bundesrat in die Hand gegeben.

\section{Zur „Integrationsverantwortung“ nach dem Lissabon-Urteil}

Ist diese in knappen Worten geschilderte „Entmachtung“ der Landesparlamente unumgänglich, ist sie verfassungsrechtlich und verfassungspolitisch akzeptabel, gewissermaßen der unverzichtbare Preis der Integrationsoffenheit des Grundgesetzes? Interessanterweise hat gerade die Entscheidung des Bundesverfassungsgerichts zum Lissabon-Vertrag ${ }^{1}$ die Frage aufgeworfen, ob jene Prozesse der „Entparlamentarisierung“ auf der Ebene der Bundesländer mit dem Grundgesetz sowie den Landesverfassungen vereinbar sind. Die in dieser Entscheidung des Bundesverfassungsgerichts wiederholt angesprochene „Integrationsverantwortung" der gesetzgebenden Organe hat bei den Anhängern einer Revitalisierung der föderalen Ordnung und des parlamentarischen Systems auf Landesebene offenbar einen Hauch von Hoffnung aufkommen lassen.

Wirft man unter diesem Aspekt tiefere Blicke in die Entscheidung des Bundesverfassungsgerichts zum Lissabon-Vertrag, mag diese Hoffnung als ziemlich trügerisch erscheinen. Zwar ist an verschiedenen Stellen davon die Rede, dass den deutschen Verfassungsorganen eine dauerhafte Integrationsverantwortung obliege, explizit sind damit aber die gesetzgebenden Organe des Bundes angesprochen. So ist von der Integrationsverantwortung vor allem in folgendem Kontext die Rede: Das Grundgesetz ermächtigt die deutschen Staatsorgane nicht, Hoheitsrechte derart zu übertragen, dass aus ihrer Ausübung heraus eigenständig weitere Zuständigkeiten begründet werden können. Es untersagt also die Übertragung der Kompetenz-Kompetenz. Das Prinzip der begrenzten Einzelermächtigung ist nicht nur ein europarechtlicher Grundsatz, sondern nimmt - ebenso wie die Pflicht der Europäischen Union, die nationale Identität der Mitgliedstaaten zu achten mitgliedstaatliche Verfassungsprinzipien auf. Das Integrationsprogramm der Europäischen Union muss deshalb hinreichend bestimmt sein. ${ }^{2}$ Sofern die Mitgliedstaaten das Vertragsrecht so ausgestalten, dass unter grundsätzlicher Fortgeltung des Prinzips der begrenzten Einzelermächtigung eine Veränderung des Vertragsrechts ohne Ratifikationsverfahren herbeigeführt werden kann, obliegt neben der Bundesregierung den gesetzgebenden Körperschaften eine besondere Verantwortung im Rahmen der Mitwirkung, die in Deutschland 
innerstaatlich den Anforderungen des Art. 23 Abs. 1 GG genügen muss: Integrationsverantwortung. ${ }^{3}$

Das Zustimmungsgesetz zu einem europäischen Änderungsvertrag und die innerstaatliche Begleitgesetzgebung müssen so beschaffen sein, dass die europäische Integration weiter nach dem Prinzip der begrenzten Einzelermächtigung erfolgt, ohne dass für die Europäische Union die Möglichkeit besteht, sich der Kompetenz-Kompetenz zu bemächtigen oder die integrationsfeste Verfassungsidentität der Mitgliedstaaten, hier des Grundgesetzes, zu verletzen. Für Grenzfälle des noch verfassungsrechtlich Zulässigen muss der deutsche Gesetzgeber mit seinen die Zustimmung begleitenden Gesetzen Vorkehrungen dafür treffen, dass die Integrationsverantwortung der Gesetzgebungsorgane sich hinreichend entfalten kann.

Es geht also unmittelbar um die innerstaatsrechtlich gebotene Mitwirkung von Bundestag und Bundesrat bei der Inanspruchnahme der Brückenklauseln, Kompetenzerweiterungs- und Flexibilitätsklauseln sowie des Notbremsemechanismus. Bei Handhabung all dieser Klauseln der neuen Unionsverträge geht es der Sache nach um eine partielle Vertragserweiterung oder Vertragsergänzung, so dass nach der innerstaatlichen Verfassungsrechtslage die gesetzgebenden Organe des Bundes wie bei einem förmlichen Ratifikationsverfahren einzuschalten sind. Es liegt geradezu in der Natur der Sache, dass in einem solchen Gedankengebäude die Landesparlamente schlicht nicht vorkommen.

\section{Wahrung des demokratisch-parlamentarischen Systems}

Gleichwohl müssen die Landesparlamente nicht in völliger Resignation zurückgelassen werden. Es gibt einen dünnen Ast, der wirklich etwas mächtiger ist als ein Strohhalm, zur Selbstrettung, denn ganz allgemein heißt es in der Lissabon-Entscheidung, dass die europäische Integration nicht zu einer Aushöhlung des demokratischen Herrschaftssystems in Deutschland führen dürfe. Den deutschen Verfassungsorganen obliege eine dauerhafte Integrationsverantwortung, die darauf gerichtet sei, bei der Übertragung von Hoheitsrechten und bei der Ausgestaltung der europäischen Entscheidungsverfahren dafür Sorge zu tragen, dass in einer Gesamtbetrachtung sowohl das politische System der Bundesrepublik Deutschland als auch das der Europäischen Union demokratischen Grundsätzen im Sinne des Art. 20 Abs. 1 und Abs. 2 in Verbindung mit Art. 79 Abs. 3 GG entspricht.

Es geht dabei nicht so sehr um die Frage, was im Einzelnen unter dem mehrdeutigen Begriff der Integrationsverantwortung zu verstehen ist, ob er sich wirklich auf die gesetzgebenden Organe der Bundesländer erstreckt, oder ob er nur im engeren, vom Bundesverfassungsgericht verwendeten Kontext zu verstehen ist. Es geht vielmehr darum, dass im europäischen Integrationsprozess, der vom Grundgesetz in Art. 23 Abs. 1 gebilligt, gefordert und im Sinne einer Staatszielbestimmung gefördert wird, die unabänderlichen Verfassungsprinzipien des demokratischen Systems und der Bundesstaatlichkeit gewahrt bleiben. Dies wird nicht zuletzt von der so genannten Ewigkeitsklausel des Art. 79 Abs. 3 GG gefordert und ist eine unüberwindbare Grenze des deutschen Verfassungsrechts im Prozess der Integration und Vergemeinschaftung. Auch wegen der so genannten Homogenitätsklausel des 
Art. 28 Abs. 1 S. 1 GG muss in den Ländern die verfassungsmäßige Ordnung unter anderem dem Demokratieprinzip entsprechen. Das bedeutet auch, dass die Verfassungen der Bundesländer so gestaltet werden beziehungsweise bestehende Vorschriften des Landesrechts so interpretiert werden müssen, dass auch in den Ländern das System der parlamentarischen Demokratie gewahrt und gesichert, den veränderten Bedingungen eines zunehmenden Prozesses der Verlagerung von Rechtsetzungsgewalt auf die Europäische Union angepasst und dem Primat des Landesgesetzgebers durch veränderte Mitwirkungsrechte des Landesparlaments bei der Unionsgesetzgebung in Bereichen ursprünglicher Landeshoheit Rechnung zu tragen ist.

Die Integrationsoffenheit des Grundgesetzes führt nach näherer Maßgabe des Art. 23 GG, der selbst nur die für den Mitgliedstaat Deutschland agierende Bundesebene betrifft und dort für eine kompensatorische Beteiligung der Organe der Bundesgesetzgebung sorgt, zu einer signifikanten Schwächung des parlamentarisch-demokratischen Systems auf der Ebene der Bundesländer, soweit es um Unionsgesetzgebung geht. Dies berührt im Falle einer europäischen Rechtsetzung auf Feldern, die nach geltender innerstaatlicher Verfassungsrechtsordnung zu den Kompetenzbereichen der Landesgesetzgebung gehören, die Grundsätze des demokratischen Systems und die Bundesstaatlichkeit, die dem Verfassungsprinzip der Unabänderlichkeit gemäß Art. 79 Abs. 3 GG unterfallen und die im Hinblick auf das demokratische System auch ausdrücklich für die Länder über die so genannte Homogenitätsklausel des Art. 28 Abs. 1 S. 1 GG verbürgt sind.

\section{Konsequenzen für das Verfassungsrecht der Bundesländer}

Die Länder haben - auf Grund zwingender bundesverfassungsrechtlicher Vorgaben - ihr Landesverfassungsrecht so auszugestalten oder - soweit dies nach geltendem Recht möglich ist - so zu interpretieren und zu handhaben, dass die geschilderten Defizite im parlamentarisch-demokratischen System in gewissem Grade kompensiert werden können. Die Zeiten sind vorbei, in denen die Verfassungsrechtslehre immer nur die Auszehrung des parlamentarischen Systems, vor allem auf der Landesebene, beklagte. Es muss nunmehr darum gehen, von Verfassung wegen kompensatorische Regelungen für die teilweise zwangsläufig eingetretenen Verluste zu fordern.

Über die in den Bundesländern, teils auf verfassungsrechtlicher Grundlage, teils auf Grund von Absprachen zwischen den Landtagen und den Landesregierungen, eingeräumten Beteiligungsrechte der Landtage hinaus wäre an von der Landesverfassung bestimmte Bindungen zu denken, die von Beschlüssen der Landtage im Hinblick auf das Abstimmungsverhalten der Landesregierungen im Bundesrat ausgehen. Von Bundesverfassung wegen sind grundsätzlich Weisungen an die Mitglieder des Bundesrates nicht ausgeschlossen, allerdings dürfen sie nach herrschender Auffassung nur von den Landesregierungen ausgehen. Gestützt wird dies auf eine ältere Entscheidung des Bundesverfassungsgerichts, wonach es unzulässig sein soll, wenn die Landesregierung in ihrem Stimmverhalten im Bundesrat durch einen Beschluss des Landtages gebunden werden soll. ${ }^{4}$ Ich halte eine solche auf Art. 51 GG gestützte Auslegung für nicht zutreffend. Inwieweit Beschlüsse des Landta- 
ges bindende Wirkung gegenüber der Landesexekutive entfalten, ist ausschließlich eine Frage des Landesverfassungsrechts. Die Länder verfügen über eine eigene Verfassungshoheit, regeln also das verfassungsrechtliche Verhältnis der Verfassungsorgane des Landes zueinander eigenständig. Bundesverfassungsrechtliche Vorgaben sind in diesem Bereich grundsätzlich unzulässig. Landespolitische und landesverfassungsrechtliche Hintergründe der Stimmabgabe im Bundesrat sind bundesverfassungsrechtlich irrelevant. ${ }^{5}$ Dass Landtagsbeschlüsse, die die Exekutive binden, mit unverzichtbaren Grundsätzen des parlamentarischen Systems und der Gewaltenteilung unvereinbar seien, so dass sie über die Homogenitätsklausel des Art. 28 Abs. 1 S. 1 GG auf der Landesebene nicht eingeführt werden könnten, erscheint gleichfalls nicht begründbar. In diesem Zusammenhang sei nur darauf hingewiesen, dass das Bundesverfassungsgericht selbst in seiner Lissabon-Entscheidung für die Bundesebene und die hier geltende Integrationsverantwortung des Bundestages solche bindenden Beschlüsse in Richtung auf das Abstimmungsverhalten im Rat ausdrücklich gefordert hat. Dies gilt etwa in den Fällen des Art. 48 Abs. 2 S. 1, des Art. 82 Abs. 3 Unterabsatz 1 S. 1 und des Art. 83 Abs. 3 Unterabsatz 1 S. 1 des Vertrages über die Arbeitsweise der Europäischen Union und die Ausübung des dort vorgesehenen Notbremsemechanismus.

Die hier angesprochenen Weisungsrechte der Landtage in Bezug auf das Abstimmungsverhalten der Landesexekutive im Bundesrat sind natürlich nicht nur in Bezug auf Rechtsetzungsakte der Union, sondern auch dann denkbar, wenn es um die Abstimmung im Bundesrat im Hinblick auf eine Subsidiaritätsrüge und die Erhebung einer Subsidiaritätsklage geht. Entsprechendes könnte gelten, wenn es darum geht, dass Landesregierungen aufgrund von Bundesrecht Normenkontrollverfahren oder andere Verfahren vor dem Bundesverfassungsgericht einzuleiten in der Lage sein sollten, welche die Gültigkeit unionsrechtlicher Rechtsakte und speziell etwa die ultra-vires-Frage auf dem Gebiet originärer Landeshoheit betreffen.

\section{Verfassungsrechtliche Notbremse trotz politischer Widerstände}

Diese Überlegungen sind weitgehend auf staatsrechtlichem Neuland angesiedelt. Rechtliche und politische Widerstände sind zweifelsohne zu erwarten. Aber es geht doch um nicht mehr, aber auch nicht um weniger als die Existenz der parlamentarischen Demokratie auf der Ebene der deutschen Bundesländer, also letztlich um deren Staatsqualität und damit um unverzichtbare Existenzbedingungen der föderalen Ordnung in der Bundesrepublik Deutschland überhaupt. Gefordert sind keine verfassungsästhetischen Einzelkorrekturen, auch wenn am Schluss bestimmte Einzelfragen der Landtagskompetenzen angesprochen worden sind. Es geht auch nicht darum, verblasstes Prestige von Landtagen, Landtagsabgeordneten und Parlamentspräsidenten aufzupolieren, sondern darum, die verfassungsrechtliche, zumindest die verfassungspolitische Notbremse zu ziehen. 